\title{
Bichiral structure of ferroelectric domain walls driven by flexoelectricity
}

\author{
P. V. Yudin,,${ }^{1, *}$ A. K. Tagantsev, ${ }^{1}$ E. A. Eliseev, ${ }^{2}$ A. N. Morozovska, ${ }^{3}$ and N. Setter ${ }^{1}$ \\ ${ }^{1}$ Ceramics Laboratory, Swiss Federal Institute of Technology (EPFL), CH-1015 Lausanne, Switzerland \\ ${ }^{2}$ Institute for Problems of Materials Science, National Academy of Sciences of Ukraine, 3, Krjijanovskogo str., UA-03142 Kiev, Ukraine \\ ${ }^{3}$ Institute of Physics, National Academy of Sciences of Ukraine, 46, pr. Nauki, UA-03028 Kiev, Ukraine
}

(Received 23 July 2012; published 3 October 2012; publisher error corrected 8 October 2012)

\begin{abstract}
The influence of flexoelectric coupling on the internal structure of neutral domain walls in the tetragonal phase of perovskite ferroelectrics is studied. The effect is shown to lower the symmetry of $180^{\circ}$ walls which are oblique with respect to the cubic crystallographic axes, while $\{100\}$ and $\{110\}$ walls stay "untouched." Being of the Ising type in the absence of the flexoelectric interaction, the oblique domain walls acquire a new polarization component with a structure qualitatively different from the classical Bloch-wall structure. In contrast to the Bloch-type walls, where the polarization vector draws a helix on passing from one domain to the other, in the flexoeffect-affected wall, the polarization rotates in opposite directions on the two sides of the wall and passes through zero in its center. Since the resulting polarization profile is invariant upon inversion with respect to the wall center, it does not break the wall symmetry, in contrast to the classical Bloch-type walls. The flexoelectric coupling lowers the domain wall energy and gives rise to its additional anisotropy, which is comparable to that conditioned by elastic anisotropy. The atomic order-of-magnitude estimates shows that the new polarization component $P_{2}$ may be comparable with spontaneous polarization $P_{s}$, thus suggesting that, in general, it is mandatory to include the flexoelectric coupling in domain wall simulations in ferroelectrics. Calculations performed for barium titanate yield the maximal value of $P_{2}$, which is much smaller than that of the spontaneous polarization. This smallness is attributed to an anomalously small value of a component of the "strain-polarization" electrostrictive tensor in this material.
\end{abstract}

DOI: 10.1103/PhysRevB.86.134102

PACS number(s): 77.80.Dj, 77.80.bn

\section{INTRODUCTION}

In light of the miniaturization of electronic devices and the achievements in nanotechnology, the question of the functionality of domain walls in ferroelectrics is an exciting issue. The current limit for nanoscale engineering is of the order of tens of nanometers. Having sizes typically of a few nanometers, domain walls offer unique properties that are not exhibited in the bulk of a ferroic sample. For example, there is experimental evidence that twin domain walls in nonferroelectric $\mathrm{CaTiO}_{3}$ possess spontaneous polarization. ${ }^{1}$ Also, ferroelectric properties are predicted in antiphase boundaries of otherwise nonferroelectric $\mathrm{SrTiO}_{3} .{ }^{2}$ Because the next logical step in the trend of miniaturization is utilization of a single domain wall as a functional element, fundamental research on the domain wall's internal structure is greatly needed. As mechanical and electric properties are strongly coupled in ferroelectrics, the domain wall structure is determined by both the electric and elastic properties of a material, and taking into account the latter may radically affect the wall structure. ${ }^{2}$ If we consider, for example, a $180^{\circ}$ domain wall, which is the junction between two oppositely poled domains, its simplest profile contains only one polarization component (Ising wall). However, it is possible that domain walls with additional polarization components are energetically favorable. A classic example is the Bloch wall, where an additional in-wall-plane polarization component arises, resulting in a helical polarization profile ${ }^{3}$ with left-handed or right-handed chirality. In uniaxial ferroelectrics with trigonal symmetry, $\mathrm{LiNbO}_{3}$ and $\mathrm{LiTaO}_{3}$, achiral two-component walls are possible, where the polarization rotates in opposite directions on the two sides of the wall and passes through zero in its center. This has been predicted by Landau theory ${ }^{4}$ and obtained by $a b$ initio calculations. ${ }^{5}$
Following the terminology introduced by Houchmandzadeh et al., ${ }^{6}$ we term such walls "bichiral."

We consider perovskite crystals with tetragonal symmetry of a ferroelectric phase. Up to now in the context of neutral ferroelectric domain walls in perovskite ferroelectrics, the description of electromechanical coupling was restricted to electrostrictive interaction, and domain walls were assumed to be either of Ising or of Bloch type. Electrostriction has a considerable influence on the stability of Ising walls and introduces anisotropy of the wall energy, ${ }^{7}$ but this effect does not introduce new features in the wall structure. Recent studies $^{8}$ revealed a considerable impact of the generalized flexoelectricity (bilinear coupling between the strain and the order parameter gradient) on the wall structure in ferroics. In this paper, we examine the effect of flexoelectricity on electrically neutral ferroelectric domain walls as a function of wall orientation. We develop Landau theory for perovskitetype ferroelectrics in the tetragonal phase, and we perform numerical calculations for $\mathrm{BaTiO}_{3}$ (BTO). It is shown that the flexoelectric effect has no impact on $90^{\circ}$ walls and on $180^{\circ}$ walls of the $\{100\}$ and $\{110\}$ orientation. At the same time, for oblique $180^{\circ}$ walls the effect leads to a wall with bichiral structure.

\section{GINSBURG-LANDAU-DEVONSHIRE THEORY}

We consider perovskite material with $m \overline{3} m$ symmetry of the parent phase. The electric displacement field is defined as $\mathbf{D}=$ $\varepsilon_{b} \mathbf{E}+\mathbf{P}$, where $\varepsilon_{b}$ is the background dielectric permittivity, $\mathbf{E}$ is the vector of the electric field, and $\mathbf{P}$ is the ferroelectric part of the polarization vector (hereafter we use the term "polarization" as shorthand). The $\mathbf{D}$ field satisfies the Poisson 
equation:

$$
\operatorname{div} \mathbf{D}=0 .
$$

The Gibbs free energy density expanded to sixth power of polarization, including gradient and flexoelectric terms, is written as follows: ${ }^{8}$

$$
\begin{aligned}
G= & A_{i j} P_{i} P_{j}+B_{i j k l} P_{i} P_{j} P_{k} P_{l}+C_{i j k l m n} P_{i} P_{j} P_{k} P_{l} P_{m} P_{n} \\
& +\frac{1}{2} D_{i j k l} \frac{d P_{i}}{d x_{j}} \frac{d P_{k}}{d x_{l}}-Q_{i j k l} \sigma_{i j} P_{k} P_{l}-\frac{1}{2} s_{i j k l} \sigma_{i j} \sigma_{k l} \\
& +\frac{1}{2} F_{i j k l}\left(\sigma_{i j} \frac{d P_{k}}{d x_{l}}-P_{k} \frac{d \sigma_{i j}}{d x_{l}}\right)
\end{aligned}
$$

where $\quad A_{i j}=a_{1} \delta_{i j}, \quad B_{i j k l}=\frac{a_{12}}{2}\left\langle\delta_{i j} \delta_{k l}\right\rangle+\left(a_{11}-a_{12}\right) g_{i j k l}^{(4)}$, and $\quad C_{i j k l m n}=\frac{a_{123}}{6}\left\langle\delta_{i j} \delta_{k l} \delta_{m n}\right\rangle+\left(a_{112}-\frac{a_{123}}{2}\right)\left\langle\delta_{i j} g_{k l m n}^{(4)}\right\rangle+$ $\left(a_{111}-a_{112}+\frac{a_{123}}{3}\right) g_{i j k l m n}^{(6)}$ are the second-, fourth-, and sixth-order dielectric stiffness tensors. With \langle\rangle we denote symmetrization with respect to interchange of indices, e.g., $\left\langle\delta_{i j} \delta_{k l}\right\rangle=\frac{1}{3}\left(\delta_{i j} \delta_{k l}+\delta_{i k} \delta_{j l}+\delta_{i l} \delta_{j k}\right)$.

$$
\begin{aligned}
D_{i j k l}= & D_{12} \delta_{i j} \delta_{k l}+D_{66}\left(\delta_{i k} \delta_{j l}+\delta_{i l} \delta_{j k}\right) \\
& +\left(D_{11}-D_{12}-2 D_{66}\right) g_{i j k l}^{(4)}
\end{aligned}
$$

is the tensor controlling the correlation effects and $\sigma_{i j}$ are the components of mechanical stress. Hereafter, the summation over repeating indices is implied, $\delta_{i j}$ is the invariant Kronecker tensor, and $g_{i j k l}^{(4)}$ and $g_{i j k l m n}^{(6)}$ are invariant tensors for the cubic symmetry. In the cubic crystallographic axes, the tensors $g_{i j k l}^{(4)}$ and $g_{i j k l m n}^{(6)}$ have the following structures: $g_{i j k l}^{(4)}=1$ if $i=j=$ $k=l$ and $g_{i j k l}^{(4)}=0$ otherwise; $g_{i j k l m n}^{(6)}=1$ if $i=j=k=l=$ $m=n$ and $g_{i j k l m n}^{(6)}=0$ otherwise.

The electrostriction tensor $Q_{i j k l}$, the compliance tensor $s_{i j k l}$, and the flexoelectric tensor $F_{i j k l}$ have the same structures as $D_{i j k l}$ :

$$
\begin{aligned}
Q_{i j k l}= & Q_{12} \delta_{i j} \delta_{k l}+\frac{Q_{66}}{4}\left(\delta_{i k} \delta_{j l}+\delta_{i l} \delta_{j k}\right) \\
& +\left(Q_{11}-Q_{12}-\frac{Q_{66}}{2}\right) g_{i j k l}^{(4)}, \\
s_{i j k l}= & s_{12} \delta_{i j} \delta_{k l}+\frac{s_{66}}{4}\left(\delta_{i k} \delta_{j l}+\delta_{i l} \delta_{j k}\right) \\
& +\left(s_{11}-s_{12}-\frac{s_{66}}{2}\right) g_{i j k l}^{(4)}, \\
F_{i j k l}= & F_{12} \delta_{i j} \delta_{k l}+\frac{F_{66}}{2}\left(\delta_{i k} \delta_{j l}+\delta_{i l} \delta_{j k}\right) \\
& +\left(F_{11}-F_{12}-F_{66}\right) g_{i j k l}^{(4)} .
\end{aligned}
$$

From the Gibbs potential (2), one obtains equations of state:

$$
\frac{\partial G}{\partial P_{i}}-\frac{d}{d x_{j}}\left(\frac{\partial G}{\partial P_{i, j}^{\prime}}\right)=0 .
$$

For mechanical stresses, we apply conditions of mechanical equilibrium:

$$
\frac{\partial \sigma_{i j}}{\partial x_{j}}=0(i, j=1-3) .
$$

In view of the presence of a stress gradient in expression (2), strain is defined via the variational derivation:

$$
\varepsilon_{i j}=-\partial G / \partial \sigma_{i j}+\frac{d}{d x_{k}}\left(\partial G / \partial \sigma_{i j, k}^{\prime}\right) .
$$

\section{STATEMENT OF THE PROBLEM FOR NEUTRAL DOMAIN WALLS}

We consider the material in the tetragonal phase, where the spatially homogeneous solution to the set of Eqs. (7) and (8) for the mechanically free sample yields six equivalent domain states $\left\{P_{s}, 0,0\right\},\left\{-P_{s}, 0,0\right\},\left\{0, P_{s}, 0\right\}$, etc. with spontaneous polarization $P_{s}=\sqrt{\frac{\sqrt{a_{11}^{2}-3 a_{111} a_{1}}-a_{11}}{3 a_{111}}} .90^{\circ}$ walls separate domains with an angle of $90^{\circ}$ (to within the clapping angle) between the polarization vectors; between oppositely poled domains, $180^{\circ}$ walls are formed. Below, we show that flexoelectricity does not affect the properties of the $\{100\}$ and $\{110\}$ walls. A condition of mechanical compatibility allows only one type of orientation for $90^{\circ}$ walls, namely $\{110\}$. Thus $90^{\circ}$ walls are not affected by flexoelectric coupling. That is why we consider only the $180^{\circ}$ walls.

\section{A. $180^{\circ}$ walls}

Electrically neutral $180^{\circ}$ walls are parallel to the $\mathbf{P}_{\mathbf{s}}$ vector. We characterize the orientation of the wall by the angle $\alpha$ between the wall normal and the $x_{3 C}$ cubic crystallographic direction, as shown in Fig. 1. Calculations are performed in the reference frame $\left(x_{1}, x_{2}, x_{3}\right)$ shown in Fig. 1, which is associated with the wall. We consider a one-dimensional (1D) problem with the polarization vector $\mathbf{P}$ and the mechanical stress tensors $\sigma_{i j}$ being dependent only on the coordinate $x_{3}$ normal to the plane of the wall. We neglect the polarization component normal to the wall as suppressed by the strong depolarizing field, so that only $P_{1}$ and $P_{2}$ components are allowed. In the new reference frame, the Gibbs energy (2) reads

$$
\begin{aligned}
G= & a_{1}\left(P_{1}^{2}+P_{2}^{2}\right)+a_{11} P_{1}^{4}+a_{22}(\alpha) P_{2}^{4}+a_{12} P_{1}^{2} P_{2}^{2}+a_{111} P_{1}^{6}+a_{222}(\alpha) P_{2}^{6} \\
& +a_{112} P_{1}^{4} P_{2}^{2}+a_{122}(\alpha) P_{2}^{4} P_{1}^{2}+\frac{D_{66}}{2}\left(\frac{\partial P_{1}}{\partial x_{3}}\right)^{2}+\frac{D_{44}(\alpha)}{2}\left(\frac{\partial P_{2}}{\partial x_{3}}\right)^{2} \\
& -Q_{11} \sigma_{1} P_{1}^{2}-Q_{22}(\alpha) \sigma_{2} P_{2}^{2}-Q_{66} \sigma_{6} P_{1} P_{2}-Q_{12}\left(\sigma_{1} P_{2}^{2}+\sigma_{2} P_{1}^{2}\right) \\
& -\frac{1}{2}\left[s_{11} \sigma_{1}^{2}+s_{22}(\alpha) \sigma_{2}^{2}\right]-s_{12} \sigma_{1} \sigma_{2}-\frac{1}{2} s_{66} \sigma_{6}^{2}+\frac{1}{2} F_{24}(\alpha)\left(\sigma_{2} \frac{\partial P_{2}}{\partial x_{3}}-P_{2} \frac{\partial \sigma_{2}}{\partial x_{3}}\right) .
\end{aligned}
$$


Here we omitted the terms that are null at $\sigma_{3}=\sigma_{4}=\sigma_{5}=0$, since as we show below in Sec. III B, these stress components do not appear in the one-dimensional case. The designations used are

$$
\begin{gathered}
a_{22}(\alpha)=a_{11}-\frac{2 a_{11}-a_{12}}{4} \sin ^{2}(2 \alpha) \\
Q_{22}(\alpha)=Q_{11}+\sin ^{2}(2 \alpha)\left(\frac{Q_{66}}{4}-\frac{Q_{11}-Q_{12}}{2}\right) \\
s_{22}(\alpha)=s_{11}+\sin ^{2}(2 \alpha)\left(\frac{s_{66}}{4}-\frac{s_{11}-s_{12}}{2}\right) \\
F_{2223}(\alpha) \equiv F_{24}(\alpha)=\frac{\sin (4 \alpha)}{4}\left(F_{66}-F_{11}+F_{12}\right) \\
\equiv F_{a} \sin (4 \alpha)
\end{gathered}
$$

$$
\begin{aligned}
a_{222}(\alpha) & =a_{111}-\frac{3 a_{111}-a_{112}}{4} \sin ^{2}(2 \alpha), \quad a_{122}(\alpha) \\
& =a_{112}-\frac{2 a_{112}-a_{123}}{4} \sin ^{2}(2 \alpha), \\
D_{44}(\alpha) & =D_{66}+\sin ^{2}(2 \alpha)\left(\frac{D_{11}-D_{12}}{2}-D_{66}\right) .
\end{aligned}
$$

The following Voigt's (matrix) notations are used:

$a_{11} \equiv a_{1}, a_{1111} \equiv a_{11}, 6 a_{1122} \equiv a_{12}, D_{1111} \equiv D_{11}, D_{1122} \equiv$ $D_{12}, \quad D_{1212} \equiv D_{66}, \quad Q_{1111} \equiv Q_{11}, \quad Q_{1122} \equiv Q_{12}, 4 Q_{1212} \equiv$ $Q_{66}, \quad s_{1111} \equiv s_{11}, \quad s_{1122} \equiv s_{12}, \quad 4 s_{1212} \equiv s_{66}, \quad F_{1111} \equiv F_{11}$, $F_{1122} \equiv F_{12}$, and $2 F_{1212} \equiv F_{66} \cdot{ }^{9}$

From (10) one obtains the equations of state for the polarization components depending only on $x_{3}$ in the form

$$
\begin{aligned}
2 a_{1} P_{1} & +4 a_{11} P_{1}^{3}+2 a_{12} P_{2}^{2} P_{1}+6 a_{111} P_{1}^{5}+4 a_{112} P_{1}^{3} P_{2}^{2}+2 a_{122}(\alpha) P_{1} P_{2}^{4} \\
- & D_{66} \frac{\partial^{2} P_{1}}{\partial x_{3}^{2}}-2\left(Q_{11} \sigma_{1}+Q_{12} \sigma_{2}\right) P_{1}-Q_{66} \sigma_{6} P_{2} \\
= & 0,2 a_{1} P_{2}+4 a_{22}(\alpha) P_{2}^{3}+2 a_{12} P_{1}^{2} P_{2}+6 a_{222}(\alpha) P_{2}^{5}+2 a_{112} P_{1}^{4} P_{2}+4 a_{122}(\alpha) P_{1}^{2} P_{2}^{3} \\
& -D_{44}(\alpha) \frac{\partial^{2} P_{2}}{\partial x_{3}^{2}}-2\left[Q_{22}(\alpha) \sigma_{2}+Q_{12} \sigma_{1}\right] P_{2}-Q_{66} \sigma_{6} P_{1}-F_{24}(\alpha) \frac{\partial \sigma_{2}}{\partial x_{3}}=0 .
\end{aligned}
$$

The boundary conditions for the polarization far from the wall are

$$
\begin{aligned}
P_{1}\left(x_{3} \rightarrow-\infty\right) & =-P_{S}, \quad P_{1}\left(x_{3} \rightarrow \infty\right) \\
& =P_{S}, \quad P_{2}\left(x_{3} \rightarrow \pm \infty\right)=0 .
\end{aligned}
$$

\section{B. Elimination of mechanical variables} free:

We consider the bulk of the domains to be mechanically

$$
\sigma_{i j}\left(x_{3} \rightarrow \pm \infty\right)=0, \quad i, j=1,2,3 .
$$

This implies, using Eqs. (2) and (9), the boundary conditions for the strain components:

$$
\varepsilon_{11}=Q_{11} P_{S}^{2}, \quad \varepsilon_{22}=Q_{12} P_{S}^{2}, \quad \varepsilon_{12}=0 .
$$

For our 1D problem, the condition of mechanical equilibrium (8) reads $\partial \sigma_{3} / \partial x_{3}=0, \partial \sigma_{4} / \partial x_{3}=0, \partial \sigma_{5} / \partial x_{3}=0$. In view of (19), this condition requires that $\sigma_{3}=\sigma_{4}=\sigma_{5}=0$ everywhere. The $1 \mathrm{D}$ character of the problem also enables us to rewrite the Saint-Venant compatibility relationships,

$$
e_{i k l} e_{j m n}\left(\partial^{2} \varepsilon_{l n} / \partial x_{k} \partial x_{m}\right)=0
$$

( $e_{i j k}$ is the Levi-Civita symbol), as

$$
d^{2} \varepsilon_{11} / d x_{3}^{2}=d^{2} \varepsilon_{12} / d x_{3}^{2}=d^{2} \varepsilon_{22} / d x_{3}^{2}=0 .
$$

The solution to Eq. (22) with boundary conditions (19) is

$$
\varepsilon_{1}\left(x_{3}\right)=Q_{11} P_{S}^{2}, \quad \varepsilon_{2}\left(x_{3}\right)=Q_{12} P_{S}^{2}, \quad \varepsilon_{6}\left(x_{3}\right)=0 .
$$

Note that this is the only possible one-dimensional solution for the elastic problem. The applicability of this solution to a stress-free finite sample is equivalent to the applicability of a one-dimensional model to a parallel plate capacitor. By applying this, we neglect the fringe elastic fields at the contact of the domain wall with the surface, which is permissible when the dimensions of the sample are much larger than the thickness of the domain wall. Note that same "partially clamped" elastic conditions are usually applied for the description of mechanical stresses in a thin ferroelectric film on a substrate. ${ }^{10}$ Under this ansatz, we solve the system of Eqs. (9) and obtain expressions for the nonzero elastic stress components in the form

$$
\begin{aligned}
& \sigma_{11} \equiv \sigma_{1}=\frac{\left(\begin{array}{l}
-F_{24}(\alpha) s_{12}\left(\partial P_{2} / \partial x_{3}\right)+\left(P_{S}^{2}-P_{1}^{2}\right)\left[Q_{11} s_{22}(\alpha)-Q_{12} s_{12}\right] \\
+P_{2}^{2}\left[Q_{22}(\alpha) s_{12}-Q_{12} s_{22}(\alpha)\right]
\end{array}\right)}{s_{22}(\alpha) s_{11}-s_{12}^{2}}, \\
& \sigma_{22} \equiv \sigma_{2}=\frac{\left(\begin{array}{l}
F_{24}(\alpha) s_{11}\left(\partial P_{2} / \partial x_{3}\right)+\left(Q_{12} s_{11}-Q_{11} s_{12}\right)\left(P_{S}^{2}-P_{1}^{2}\right) \\
+P_{2}^{2}\left[-s_{11} Q_{22}(\alpha)+Q_{12} s_{12}\right]
\end{array}\right)}{s_{22}(\alpha) s_{11}-s_{12}^{2}}, \\
& \sigma_{12} \equiv \sigma_{6}=-\frac{Q_{66}}{s_{66}} P_{1}\left(x_{3}\right) P_{2}\left(x_{3}\right) .
\end{aligned}
$$




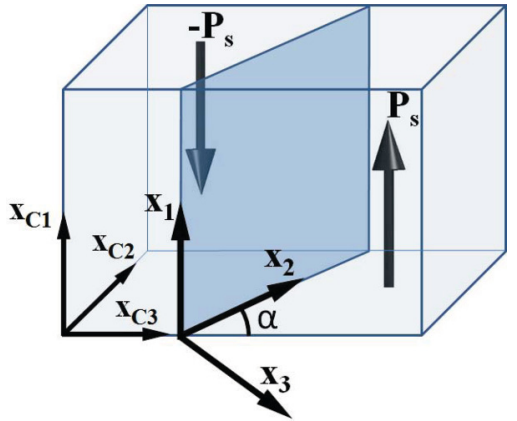

FIG. 1. (Color online) Orientation of the $180^{\circ}$ domain wall and reference frames used.

Equations (24) and (17) form a full set of equations to define the polarization profile.

\section{ANALYSIS OF EQUATIONS AND ESTIMATES}

First, as a benchmark, let us consider the set (17) and (24) without the flexoelectric effect [with $F_{24}(\alpha)$ set to zero]. One can check that at $F_{24}(\alpha)=0$, the set (17) and (24) has a singlecomponent solution, as Eq. (17b) can be satisfied with $P_{2}=0$. This Ising solution may be either stable or unstable, ${ }^{11}$ in the latter case, a Bloch wall profile is observed, with $P_{2}$ being an even function of $x_{3}$. We consider the first case, where the Ising profile is stable if the flexoelectric effect is neglected.

Since $F_{24}(\alpha)$ is proportional to $\sin (4 \alpha)(14)$, the flexoelectric effect does not reveal itself for $\{100\}(\alpha=0)$ and $\{110\}$ ( $\alpha=\pi / 4$ ) wall orientations. Hence, these walls remain Ising with the flexoelectric effect "switched on." For all the other wall orientations (hereafter referred to as oblique walls), the flexoelectric coupling inevitably leads to the appearance of an additional polarization component. Indeed, substitution of (24) into Eq. (17b) introduces an additional coupling between the polarization components. As a result, the one-component solution for the polarization profile (with $P_{2}=0$ ) is no longer available.

It is instructive to note that $\{110\}$-oriented $90^{\circ}$ domain walls do not "feel" the flexoelectric coupling for the same reason. The statement of the problem for $90^{\circ}$ walls is the same, but with boundary conditions

$$
\begin{aligned}
P_{1}\left(x_{3} \rightarrow \pm \infty\right) & =0, \quad P_{2}\left(x_{3} \rightarrow-\infty\right)=-P_{s} / \sqrt{2}, \\
P_{2}\left(x_{3} \rightarrow \infty\right) & =P_{s} / \sqrt{2}
\end{aligned}
$$

instead of (18) and with $P_{3}\left(x_{3}\right)=P_{s} / \sqrt{2}$. One can carry out the same analysis as we have done for $180^{\circ}$ walls and check that flexoelectricity produces no nonzero terms in the equations of state for the polarization vector. Thus the flexoelectric effect does not affect either $90^{\circ}$ walls or $\{100\}$ - and $\{110\}$-oriented $180^{\circ}$ walls. In contrast, oblique $180^{\circ}$ walls inevitably acquire the new polarization component $P_{2}$.

\section{A. Estimation of the magnitude of the flexoelectric-effect-induced $\boldsymbol{P}_{\mathbf{2}}$ component}

To roughly estimate the magnitude of the $P_{2}$ component induced by the flexoelectric effect, we consider the linearized Eq. (17b), where we use for $P_{1}$ the profile of an Ising wall not perturbed by flexoelectric coupling. This approximation is fully valid in the vicinity of $\{100\}$ and $\{110\}$ wall orientations, where flexoelectric coupling is small. Neglecting $P_{2}\left(x_{3}\right)$ with respect to $P_{1}\left(x_{3}\right)$ in Eq. (17a), we obtain the standard wall profile, $^{12}$

$$
\begin{aligned}
P_{1}^{2}= & P_{S}^{2} \sinh ^{2}\left(x_{3} / R_{c}\right)\left[\cosh ^{2}\left(x_{3} / R_{c}\right)+A\right]^{-1} \\
R_{c}= & \sqrt{\frac{D_{66}}{P_{S}^{2}\left(2 \tilde{a}_{11}+6 a_{111} P_{S}^{2}\right)}}, \\
A= & \frac{2 a_{111} P_{S}^{2}}{2 \tilde{a}_{11}+4 a_{111} P_{S}^{2}}, \\
\tilde{a}_{11} \equiv & a_{11} \\
& +\frac{Q_{12}\left(Q_{12} s_{11}-Q_{11} s_{12}\right)+Q_{11}\left[Q_{11} s_{22}(\alpha)-Q_{12} s_{12}\right]}{2 s_{22}(\alpha) s_{11}-s_{12}^{2}} .
\end{aligned}
$$

Linearizing the set of Eqs. (24) and (17b) with respect to $P_{2}$, we obtain

$$
\begin{aligned}
\left(2 \tilde{a}_{1}+\right. & \left.2 \tilde{a}_{12} P_{1}^{2}+2 a_{112} P_{1}^{4}\right) P_{2}-\tilde{D}_{44}(\alpha) \frac{\partial^{2} P_{2}}{\partial x_{3}^{2}} \\
= & f(\alpha) \frac{\partial\left(P_{s}^{2}-P_{1}^{2}\right)}{\partial x_{3}}, \\
\tilde{a}_{1}= & a_{1}+\Theta P_{s}^{2}, \\
\tilde{a}_{12}= & a_{12}+\frac{Q_{66}}{2 s_{66}}-\Theta, \\
\Theta= & \frac{s_{12}\left[Q_{12}^{2}-Q_{11} Q_{22}(\alpha)\right]-Q_{12}\left[Q_{22} s_{11}-Q_{11} s_{22}(\alpha)\right]}{2 s_{22}(\alpha) s_{11}-s_{12}^{2}}, \\
& \tilde{D}_{44}(\alpha) \\
\equiv D_{44}(\alpha)+\frac{F_{24}(\alpha)^{2} s_{11}}{s_{22}(\alpha) s_{11}-s_{12}^{2}}, & (27 \mathrm{~b}) \\
f(\alpha)= & \frac{\left(Q_{12} s_{11}-Q_{11} s_{12}\right)}{s_{22}(\alpha) s_{11}-s_{12}^{2}} F_{24}(\alpha),
\end{aligned}
$$

with $P_{1}$ coming from (26).

Because $P_{1}$ is an odd function, the symmetry of Eq. (27) allows an odd solution for the $P_{2}\left(x_{3}\right)$ component. As we confirm with the numerical calculations below, the odd solution is stable, meaning, in particular, $P_{2}=0 ; d P_{2} / d x_{3} \neq 0$ at $x_{3}=0$. Hence, in the vicinity of $x_{3}=0$, the main contribution to the Landau energy is due to the gradient term, which allows us to derive an approximate solution by neglecting the linear term with respect to $P_{2}$. We also set $A \rightarrow 0$ in Eq. (30) for simplicity. Upon simplifying in this way, in the vicinity of $x_{3}=0$, Eq. (27) transforms into

$$
\tilde{D}_{44}(\alpha) \frac{\partial^{2} P_{2}}{\partial x_{3}^{2}}=f(\alpha) \frac{\partial\left(P_{1}^{2}-P_{s}^{2}\right)}{\partial x_{3}} .
$$

The first integral of Eq. (28) is

$$
\tilde{D}_{44}(\alpha) \frac{\partial P_{2}}{\partial x_{3}}=f(\alpha)\left[\left(P_{1}^{2}-P_{S}^{2}\right)+C_{0}\right] \text {. }
$$


We set $C_{0}=0$ to prevent the linear increase of $P_{2}$ at $x_{3} \rightarrow$ $\pm \infty$. Taking into account that $P_{2}(0)=0$, we obtain the solution in the form

$$
\begin{aligned}
P_{2}\left(x_{3}\right) & =\frac{f(\alpha)}{\tilde{D}_{44}(\alpha)} \int_{0}^{x_{3}}\left(P_{1}^{2}-P_{S}^{2}\right) d x \\
& \approx-\frac{f(\alpha)}{\tilde{D}_{44}(\alpha)} P_{S}^{2} R_{c} \tanh \left(\frac{x_{3}}{R_{c}}\right) .
\end{aligned}
$$

The solution (30) does not satisfy the boundary conditions $P_{2}\left(x_{3} \rightarrow \pm \infty\right)=0$. This means that the other term that we do not take into account in (28) is responsible for the decay of $P_{2}$ in the domains. However, the value $P_{m}=P_{2}\left(x_{3}=\infty\right)$ from (30) may be used for an estimation of the amplitude of $P_{2}$. Reverting to the initial designations, we obtain $P_{m}$ in the form

$$
\begin{gathered}
P_{m}=\frac{F_{24}(\alpha)}{D_{44}(\alpha)\left(s_{22}(\alpha)-\frac{s_{12}^{2}}{s_{11}}\right)+F_{24}(\alpha)^{2}} \\
\quad \times\left(Q_{12}-Q_{11} \frac{s_{12}}{s_{11}}\right) P_{S}^{2} R_{c} .
\end{gathered}
$$

We rewrite it in dimensionless form:

$$
\begin{gathered}
\frac{P_{m 0}}{P_{s}}=\frac{F_{0}}{\sqrt{D_{66} s_{11}}} \frac{Q_{11}}{\sqrt{2 \tilde{a}_{11} s_{11}}}\left(\frac{Q_{12}}{Q_{11}}-\frac{s_{12}}{s_{11}}\right) \Gamma(\alpha) \\
\Gamma(\alpha)=\frac{\sin (4 \alpha) D_{66} / D_{44}(\alpha)}{\left(1+\frac{F_{24}(\alpha)^{2}}{D_{44}(\alpha)\left(s_{22}(\alpha)-\frac{s_{12}^{2}}{s_{11}^{2}}\right)}\right)\left[1-\frac{s_{12}^{2}}{s_{11}^{2}}+\sin ^{2}(2 \alpha)\left(\frac{s_{66}}{4 s_{11}}+\frac{s_{12}}{2 s_{11}}-\frac{1}{2}\right)\right]} .
\end{gathered}
$$

Let us analyze expression (32). The factor $\frac{F_{0}}{\sqrt{D_{66} s_{11}}}$ is of the order of unity according to atomic estimates. The applicability of atomic estimates for the evaluation of the flexoelectric tensor in perovskite ferroelectrics is supported by experimental evidence of rather strong flexoelectric coupling in these materials. ${ }^{13}$ The factor $\frac{Q_{11}}{\sqrt{\tilde{a}_{11} s_{11}}}$, which is responsible for the sound velocity change near the ferroelectric phase transition, is also of the order of unity in ferroelectrics with strong electromechanical coupling. ${ }^{14}$ Landau theory does not require that the dimensionless factors $\frac{Q_{12}}{Q_{11}}-\frac{s_{12}}{s_{11}}$ and $\Gamma(\alpha)$ are small compared to unity, so that for rough estimation they may be taken as unity. Thus from formula (32), the flexoelectric-driven component $P_{2}$ is expected to be of the same order as $P_{s}$.

It is instructive to specify the above estimates for BTO crystal, for which, further in the paper, we will present numerical simulations for the polarization profile of the domain boundary. For BTO, the factor $\frac{Q_{12}}{Q_{11}}-\frac{s_{12}}{s_{11}}$ appears to about $1 / 15$. The smallness of this factor might be considered purely accidental in view of the seemingly different physical phenomena behind elasticity and electrostriction. However, this smallness is of a purely electromechanical nature. To see this, we rewrite the factor $Q_{12} / Q_{11}-s_{12} / s_{11}$ in terms of the "strain-polarization" electrostriction tensor $q_{i j}$ linked with $Q_{i j}$ by the relationship $Q_{i j}=s_{j l} q_{i l}$. The tensor $q_{i j}$ can be considered to be a primary material parameter since it is directly controlled by the lattice mechanics of the crystal. Because we are interested in perovskites where the Poisson ratio $-s_{12} / s_{11}$ is typically about 0.3 , we readily find $Q_{12} / Q_{11}-s_{12} / s_{11} \simeq q_{12} / q_{11}\left\{0.5 /\left[1-0.6\left(q_{12} / q_{11}\right)\right]\right\}$. Thus we see that the smallness of the factor $Q_{12} / Q_{11}-s_{12} / s_{11}$ is conditioned upon the fact that, in metal-oxide ferroelectric perovskites, the ratio $q_{12} / q_{11}$ is typically very small compared to unity. In particular, for $\mathrm{SrTiO}_{3}, q_{12} / q_{11}=-0.086,{ }^{15}$ and in $\mathrm{Pb}\left(\mathrm{ZrO}_{3}\right)_{1-x}\left(\mathrm{TiO}_{3}\right)_{x}(\mathrm{PZT}),\left|q_{12} / q_{11}\right|<0.05$ for $x \in(0.6,1)$ ( $q_{11}$ and $q_{12}$ are recalculated using Refs. 16 and 17). However, there are materials where $q_{12}$ is not small with respect to $q_{11}$. For example, in $\mathrm{KNbO}_{3}, q_{12} / q_{11}=-0.37$, and in tetragonal PZT near the morphotropic boundary, $q_{12} / q_{11} \sim$ -0.5 (Refs. 16 and 17). There are no data available on the flexoelectric tensor coefficients in the latter materials, but one can expect relatively large domain-wall energy anisotropy and flexoelectric-effect-induced polarization components there due to the elevated $q_{12} / q_{11}$ ratio.

For BTO, the estimate for the amplitude of the second polarization component is also affected by the exceptionally high anisotropy of the correlation energy $\left(D_{11} / D_{66} \simeq 25\right)$. This occurs via the factor $\frac{D_{66}}{D_{44}(\alpha)}$ in estimate (32). Thus we conclude that because it is simultaneously affected by two small factors, the maximal value of the second polarization component in $180^{\circ}$ walls in BTO is expected to be two orders of magnitude smaller than $P_{s}$. We would like to stress that there is no reason to expect anomalously small values of this component in ferroelectrics in general.

\section{NUMERICAL RESULTS AS APPLIED TO BARIUM TITANATE}

To be more specific, we analyze the impact of flexoelectric coupling on the structure of a domain wall in ferroelectrics for the case of the tetragonal phase in classical perovskite ferroelectric $\mathrm{BaTiO}_{3}$ at room temperature. Since the problem is not analytically tractable, we do it numerically using the thermodynamic parameters of this crystal. Since the experimental values of the components of the flexocoupling tensor $F_{i j k l}$ are not currently available, we use in our calculations the $F_{i j k l}$ tensor evaluated from the data of $a b$ initio calculations for $\mathrm{Ba}_{0.5} \mathrm{Sr}_{0.5} \mathrm{TiO}_{3}$ (BST) from Ref. 18. In the case where the results are found to be critical to the exact value of this tensor, we vary this tensor value to cover different possible outcomes. The parameters used for the simulations are listed in Table I.

The profiles for $P_{1}$ and $P_{2}$ polarization components obtained from a numerical solution to Eqs. (17) and (24) are shown in Fig. 2. We use (18) as the boundary conditions, with 
TABLE I. Free-energy coefficients for bulk ferroelectric $\mathrm{BaTiO}_{3}$ (from Refs. 19, 20, 10, and 18).

\begin{tabular}{|c|c|c|}
\hline Parameters & Values & Refs. and Notes \\
\hline$a_{1}\left(\mathrm{C}^{-2} \mathrm{~m} \mathrm{~J}\right)$ & $a_{1}=3.34(T-381) \times 10^{5}$ & Ref. 19 \\
\hline$a_{i j}\left(\mathrm{C}^{-4} \mathrm{~m}^{5} \mathrm{~J}\right)$ & $\begin{array}{c}a_{11} \simeq 4.69(T-393) \times 10^{6}-2.02 \times 10^{8} \\
a_{12} \simeq 3.230 \times 10^{8}\end{array}$ & Ref. 19 \\
\hline$a_{i j k}\left(\mathrm{C}^{-6} \mathrm{~m}^{9} \mathrm{~J}\right)$ & $\begin{array}{c}a_{111} \simeq-5.52(T-393) \times 10^{7}+2.76 \times 10^{9} \\
a_{112}=4.47 \times 10^{9}, \quad a_{123}=4.91 \times 10^{9}\end{array}$ & Ref. 19 \\
\hline$D_{i j}^{(u)}\left(\mathrm{C}^{-2} \mathrm{~m}^{3} \mathrm{~J}\right)$ & $\begin{array}{c}D_{11}^{(u)}=5.1 \times 10^{-10}, \quad D_{12}^{(u)} \simeq-0.2 \times 10^{-10} \\
D_{66}^{(u)} \simeq 0.2 \times 10^{-10}\end{array}$ & Ref. 20 \\
\hline$Q_{i j}\left(\mathrm{C}^{-2} \mathrm{~m}^{4}\right)$ & $Q_{11}=0.11, \quad Q_{12} \simeq-0.043, \quad Q_{66}=0.059$ & Ref. 10 \\
\hline$s_{i j}\left(10^{-12} \mathrm{~Pa}^{-1}\right)$ & $s_{11}=8.3, s_{12} \simeq-2.7, s_{66}=9.24$ & Ref. 10 \\
\hline$f_{i j k l}^{(\mathrm{BST})}(\mathrm{V})$ & $f_{11}=5.12, \quad f_{12}=3.32, \quad f_{66}=0.045$ & Ref. 18 \\
\hline$F_{i j k l}\left(10^{-11} \mathrm{C}^{-1} \mathrm{~m}^{3}\right)$ & $\begin{array}{c}F_{11}=2.46, F_{12}=0.48, F_{66}=0.05 \\
F_{a}^{(\mathrm{BST})}=F_{66}-F_{11}+F_{12} \approx-1.93\end{array}$ & $\begin{array}{l}\text { Recalculated using relationship } F_{\alpha \gamma}=f_{\beta \gamma} s_{\beta \gamma} ; \\
f_{\alpha \gamma} \text { taken from Ref. } 18\end{array}$ \\
\hline$D_{i j k l}^{(\sigma)} \equiv D_{i j k l},\left(\mathrm{C}^{-2} \mathrm{~m}^{3} \mathrm{~J}\right)$ & $\begin{array}{c}D_{11}^{(\sigma)} \equiv D_{11}=3.52 \times 10^{-10}, \quad D_{12}^{(\sigma)} \equiv D_{12}=-1.24 \times 10^{-10} \\
D_{66}^{(\sigma)} \equiv D_{66}=0.2 \times 10^{-10}\end{array}$ & recalculated using $D_{\alpha \gamma}^{(\sigma)}=D_{\alpha \gamma}^{(u)}-f_{\alpha \beta} F_{\beta \gamma}$ \\
\hline
\end{tabular}

an additional condition of the vanishing of spatial derivatives of all variables at infinity. The $P_{1}$ profile is perfectly described by formula (26); the difference conditioned upon the flexoelectric effect is within the linewidth of the plot for any angle $\alpha$. The maximal value of $P_{2}$ is as small as $6 \times 10^{-2} \mu \mathrm{C} / \mathrm{cm}^{2}$, which is $\sim P_{S} / 300$. One can see from Fig. 2 that the width of the domain wall with respect to $P_{2}$ is a few times larger than that with respect to $P_{1}$. This is a consequence of the small ratio of correlation lengths $\frac{r\left(P_{1}\right)}{r\left(P_{2}\right)}=\frac{\sqrt{D_{66}}}{\sqrt{D_{44}(\alpha)}}$, which results from $D_{11} / D_{66} \simeq 25$. In the limit $P_{2} \ll P_{S}$ and $r\left(P_{1}\right) \ll r\left(P_{2}\right)$, an approximate analytical solution for the wall profile can also be developed, which is given in Appendix A.

The dependence of the maximal polarization $P_{2}\left(x_{3}\right)$ on the angle $\alpha$ is shown in Fig. 3. The anisotropy may be understood from expression (31) as an interplay between the angular dependence of the flexoelectric factor $F_{24}(\alpha)$ and that of the gradient-energy factor $D_{44}(\alpha)$.

Inspection of the results of the simulations reveals that flexoelectric coupling can lead to the formation of a bichiral

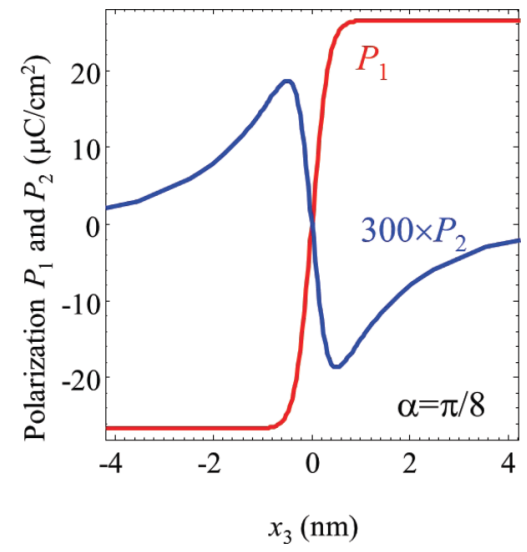

FIG. 2. (Color online) $P_{1}\left(x_{3}\right)$ and $P_{2}\left(x_{3}\right)$ profiles across a $180^{\circ}$ domain wall calculated for the parameters of $\mathrm{BaTiO}_{3}$ at room temperature given in Table I. The $P_{1}\left(x_{3}\right)$ profile is practically independent of the angle $\alpha$ between the wall and a cubic crystallographic direction. The $P_{2}\left(x_{3}\right)$ profile is calculated for $\alpha=\pi / 8$. polarization profile, which has never been obtained for $180^{\circ}$ domain walls (see Fig. 4) in tetragonal ferroelectrics. The polarization rotates in the boundary, but the latter does not acquire chirality. The senses of the polarization rotation on the two sides of the boundary are opposite, with the polarization passing though zero at its center. As a result, the polarization profile of the modified wall remains invariant with respect to the inversion about the wall center, in contrast to the Bloch-type wall. Following the terminology introduced by Houchmandzadeh et $a .^{6}$ such a wall can be classified as bichiral. One should note that the rough approximate solution (30) qualitatively correctly reproduced the bichirality of the wall.

\section{A. Free-energy calculations}

In this subsection, we study the anisotropy of DW energy induced by the flexoelectric effect. The expression for the free energy density $\Phi$ may be obtained from the Gibbs potential $G$ by Legendre transformation $\Phi=G+\sigma_{i} \varepsilon_{i}$. The wall energy
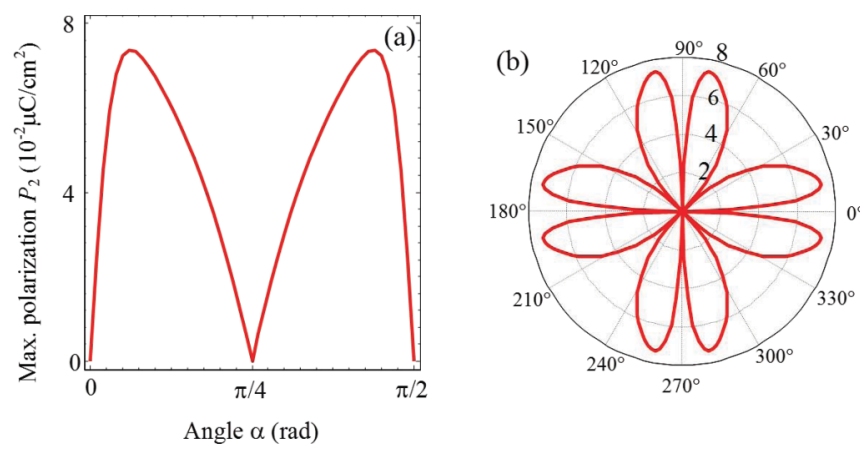

FIG. 3. (Color online) Maximal absolute value of the second polarization component in the wall as a function of the angle $\alpha$ between it and a cubic crystallographic direction calculated for the parameters of $\mathrm{BaTiO}_{3}$ at room temperature given in Table I. (a) Plot in one quadrant; (b) polar plot. 
per unit area, $E_{W}$, is then given by the integral $E_{W}=\int_{-\infty}^{+\infty}\left[\Phi\left(x_{3}\right)-\Phi_{\infty}\right] d x_{3}$, where $\Phi_{\infty}$ is the energy density at $x_{3} \rightarrow \pm \infty$. From (10), with the help of (23) and (24), we derive the following expression for the wall free energy:

$$
\begin{aligned}
E_{W}= & \int_{-\infty}^{\infty}\left\{a_{1}\left(P_{1}^{2}-P_{S}^{2}+P_{2}^{2}\right)+a_{11}\left(P_{1}^{4}-P_{S}^{4}\right)+a_{22}(\alpha) P_{2}^{4}+a_{12} P_{1}^{2} P_{2}^{2}+a_{111}\left(P_{1}^{6}-P_{S}^{6}\right)+a_{222}(\alpha) P_{2}^{6}+a_{112} P_{1}^{4} P_{2}^{2}\right. \\
& +a_{122}(\alpha) P_{2}^{4} P_{1}^{2}+\frac{D_{66}}{2}\left(\frac{\partial P_{1}}{\partial x_{3}}\right)^{2}+\frac{1}{2}\left(D_{44}(\alpha)+\frac{F_{24}^{2}(\alpha) s_{11}}{s_{22}(\alpha) s_{11}-s_{12}^{2}}\right)\left(\frac{\partial P_{2}}{\partial x_{3}}\right)^{2} \\
& +F_{24}(\alpha) \frac{Q_{12} s_{11}-Q_{11} s_{12}}{s_{22}(\alpha) s_{11}-s_{12}^{2}}\left(P_{S}^{2}-P_{1}^{2}\right) \frac{\partial P_{2}}{\partial x_{3}}+\frac{\left[Q_{11}\left(P_{S}^{2}-P_{1}^{2}\right)-Q_{12} P_{2}^{2}\right]^{2}}{2 s_{11}} \\
& \left.+\frac{\left[\left(Q_{12} s_{11}-Q_{11} s_{12}\right)\left(P_{S}^{2}-P_{1}^{2}\right)+P_{2}^{2}\left(-s_{11} Q_{22}(\alpha)+Q_{12} s_{12}\right)\right]^{2}}{2 s_{11}\left[s_{22}(\alpha) s_{11}-s_{12}^{2}\right]}+\frac{Q_{66}^{2}}{2 s_{66}} P_{1}^{2} P_{2}^{2}\right\} d x_{3} .
\end{aligned}
$$

Using the coordinate dependences obtained numerically, and using this relationship, we calculate the energy of the bichiral wall as a function of the angle $\alpha$ plotted in Fig. 5. It is seen from this figure that the anisotropy of the wall energy is extremely weak. However, it is worth looking closer at the fine stricture of the anisotropic part of the wall energy, specifically to decompose it to the parts independent of and dependent on the flexoelectric coupling $E_{W A}^{(s)}$ and $E_{W A}^{(F)}$, respectively. Such decomposition is presented in Fig. 6 for the case in which for the flexoelectric coefficients of BTO, we use those obtained by ab initio calculations for BST. As we can see, there is an essential difference between the $E_{W A}^{(s)}$ and $E_{W A}^{(F)}$ angular dependences. $E_{W A}^{(s)}$ is minimal at $\alpha=0$, maximal at $\alpha=\pi / 4$, and monotonic in the interval $(0, \pi / 4)$, while $E_{W A}^{(F)}$ is maximal in both $\alpha=0$ and $\alpha=\pi / 4$, and has a minimum in an intermediate point, around $\alpha=\pi / 10$. Remarkably, for small angles $\alpha$ both contributions are comparable, the flexoelectric one being a bit smaller. However, if we take the flexoelectric factor $F_{a}$ to be two times as large as that for BST, the flexoelectric contribution becomes dominating at small $\alpha$. This leads to a qualitative change of the anisotropy of the wall energy: flexoelectric coupling results in splitting each of the energy minima (at $\alpha=0$ and $\alpha=\pi / 2$ ) into two; see Fig. 7.
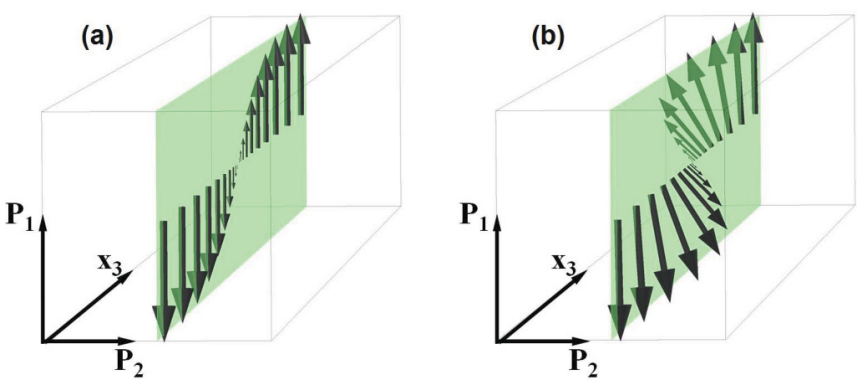

FIG. 4. (Color online) Schematic of the structures of neutral $180^{\circ}$ domain walls addressed in the paper. (a) The Ising-type structure occurring when the wall is normal to the cubic crystallographic directions or/and when the flexoelectric coupling is isotropic or neglected; (b) the bichiral structure occurring for the oblique orientation of the wall provided that the flexoelectric coupling is anisotropic.
The results of the numerical calculations can be elucidated using some analytical relationships. First, the contribution $E_{W A}^{(s)}$ can be readily presented in a simple form,

$$
\begin{aligned}
E_{W A}^{(s)}= & \frac{Q_{11}^{2}}{2 s_{11}}\left(\frac{Q_{12}}{Q_{11}}-\frac{s_{12}}{s_{11}}\right)^{2} \frac{s_{11}^{2}}{s_{22}(\alpha) s_{11}-s_{12}^{2}} \\
& \times \int_{-\infty}^{\infty}\left(P_{s}^{2}-P_{1}^{2}\right)^{2} d x_{3},
\end{aligned}
$$

which is consistent with the results by Dvorak and Janovec. ${ }^{7}$ As was recognized by these authors (and as is clear from this expression), the angular dependence of this contribution is conditioned upon that of the elastic compliance. The flexoelectricity-conditioned contribution can be evaluated taking into account that in the case of BTO, $P_{2} \ll P_{s}$ and $r\left(P_{1}\right) \ll$ $r\left(P_{2}\right)$. This enables us to keep among $P_{2}$-containing terms in (33) only $d P_{2} / d x_{3}$ (one can check that terms containing $P_{2}^{2}$ are smaller by a factor $\left.\frac{D_{66}}{D_{44}(\alpha)}\right)$ while using (29) as an approximate relationship for $d P_{2} / d x_{3}$ to get

$$
E_{W A}^{(F)} \approx E_{W A}^{(s)} \frac{2 F_{24}^{2}(\alpha) s_{11}}{\tilde{D}_{44}(\alpha)\left[s_{22}(\alpha) s_{11}-s_{12}^{2}\right]} .
$$

It is clear from this relationship that the smallness of both anisotropic contributions to the wall energy is controlled by the square of the same factor, $Q_{12} / Q_{11}-s_{12} / s_{11} \simeq 1 / 15$, which was already recognized as being responsible for the smallness

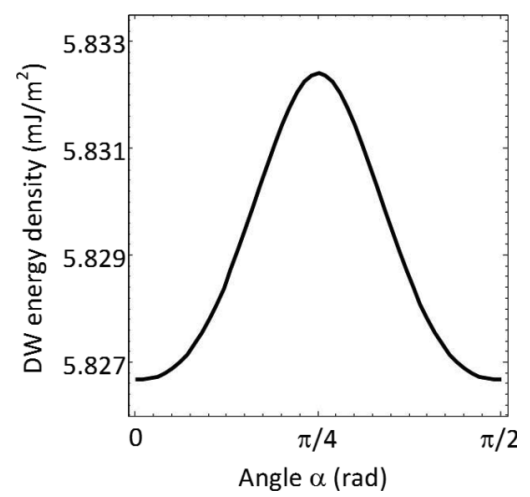

FIG. 5. Energy of a bichiral wall as a function of the angle $\alpha$ between it and a cubic crystallographic direction. Parameters used in the calculations are listed in Table I at room temperature, $T=293 \mathrm{~K}$. 


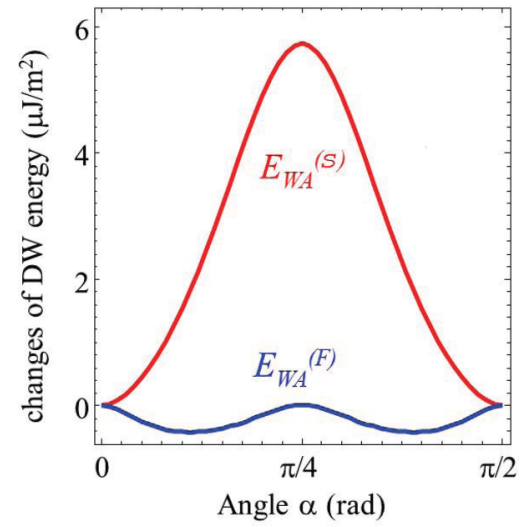

FIG. 6. (Color online) Comparison of the anisotropic contributions to the energy of bichiral wall as functions of the angle $\alpha$ between it and a cubic crystallographic direction. $E_{W A}^{(s)}$ is the contribution solely controlled by the elastic anisotropy. $E_{W A}^{(F)}$ is the contribution due to the anisotropy of the flexoelectric coupling. The calculations are done for the parameters listed in Table I at room temperature, $T=293 \mathrm{~K}$.

of the second polarization component. As for the shape of the angular dependence of the wall energy, it is conditioned upon an interplay among the elastic anisotropy, the anisotropy of the correlation energy, and that of the flexoelectric coupling.

\section{CONCLUSIONS}

The presented analysis demonstrates that the flexoelectric coupling once it is anisotropic (at $F_{a}=F_{11}-F_{12}-F_{66}=$ 0 , all effects addressed in the paper disappear) results in the formation of $180^{\circ}$ ferroelectric domain walls where the polarization rotates but the wall does not acquire chirality. Following the terminology introduced by Houchmandzadeh et al., ${ }^{6}$ such a wall can be classified as bichiral. In contact with the Bloch-type walls, the appearance of the second polarization component in bichiral walls dose not brake the wall symmetry

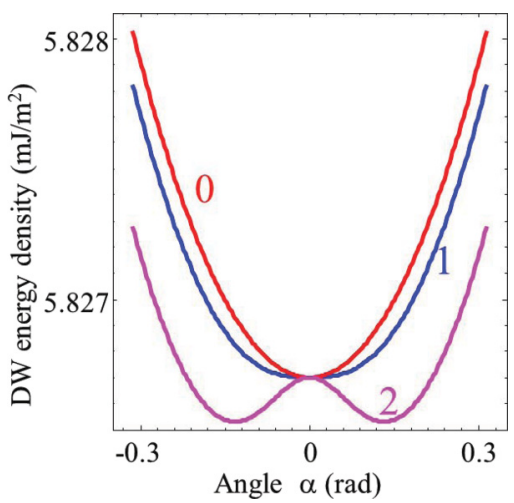

FIG. 7. (Color online) Energy of a bichiral domain wall as a function of its orientation for different values of the flexoelectric coupling calculated for the parameters of BTO (Table I) at room temperature, $T=293$. The curves labeled with 0,1 , and 2 correspond to the values of the anisotropic part of the flexoelectric coupling $F_{a}$ equal to $0, F_{a}^{(\mathrm{BST})}$, and $2 F_{a}^{(\mathrm{BST})}$, where $F_{a}^{(\mathrm{BST})}$ is the value of the flexoelectric coefficient for BST recalculated using the results of $a b$ initio from Ref. 18 (see Table I). with respect to the spatial inversion. In addition, depending on the value of $F_{a}$, the flexoelectric coupling can lead to a doubling of the number of energetically favorable orientations of the walls. Order-of-magnitude estimates show that, in general, the effects driven by the flexoelectric coupling can be appreciable. However, for the thermodynamic parameters of barium titanate, the calculations performed show that the amplitude of the second component is expected to be smaller than $1 \%$ of the spontaneous polarization, while the modulation of the wall energy (as a function of its orientation) is found to be even smaller. The numerical smallness of these effects is shown to be mainly conditioned by that of the $q_{12}$ component of the electrostriction "strain-polarization" tensor in this ferroelectric. We would like to stress that there is no reason to expect the flexoelectricity-induced features of $180^{\circ}$ ferroelectric domain walls addressed in this paper to be anomalously small in ferroelectrics in general.

\section{ACKNOWLEDGMENTS}

E.A.E and A.N.M. are thankful to NAS Ukraine. P.V.Y., A.K.T., and N.S. acknowledge the Swiss National Science Foundation for financial support.

\section{APPENDIX: APPROXIMATE ANALYTICAL SOLUTION FOR THE WALL PROFILE}

Let us derive an analytical solution for the polarization profile in the approximation $P_{2} \ll P_{S} ; r\left(P_{1}\right) \ll r\left(P_{2}\right)$. We solve Eq. (27) taking $P_{1}^{2} \rightarrow P_{S}^{2}$ everywhere except the derivative $\frac{\partial \sigma_{2}}{\partial x_{3}}$, which is the driving force for the $P_{2}$ appearance. This approximation is valid far from the wall center where $P_{1} \approx P_{S}$, and in the wall center where the gradient term is dominating over the term distorted by $P_{1}^{2} \rightarrow P_{S}^{2}$.

The solution to the second-order differential equation with constant coefficients, coordinate-dependent inhomogeneity, and boundary conditions (18) could be written using the Green

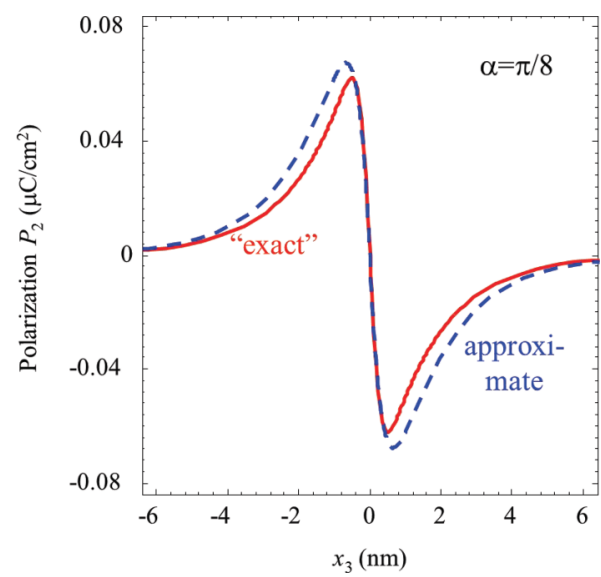

FIG. 8. (Color online) $P_{2}\left(x_{3}\right)$ profile near $180^{\circ} \mathrm{DW}$ in $\mathrm{BaTiO}_{3}$ calculated for angle $\alpha=\pi / 8$ and parameters from Table I and room temperature. Approximate analytical expression (A5) (dashed curves) and numerical simulations (solid curve) are shown. 
function method. ${ }^{21}$ Rewriting Eq. (27) for $P_{2}$ in the form

$$
\begin{aligned}
P_{2}-r^{2} \frac{\partial^{2} P_{2}}{\partial x_{3}^{2}}= & \frac{F_{24}(\alpha)}{2 a_{1}+\left[2 a_{12}+\left(Q_{66}^{2} / s_{66}\right)\right] P_{S}^{2}+2 a_{112} P_{S}^{4}} \\
& \times \frac{\left(Q_{12} s_{11}-Q_{11} s_{12}\right)}{s_{22}(\alpha) s_{11}-s_{12}^{2}} \frac{\partial\left(P_{S}^{2}-P_{1}^{2}\right)}{\partial x_{3}},
\end{aligned}
$$

where

$$
r=\sqrt{\frac{\tilde{D}_{44}(\alpha)}{2 a_{1}+\left[2 a_{12}+\left(Q_{66}^{2} / s_{66}\right)\right] P_{S}^{2}+2 a_{112} P_{S}^{4}}},
$$

one could readily ${ }^{22}$ find that

$$
\begin{aligned}
P_{2}\left(x_{3}\right)= & \frac{1}{2 r} \int_{-\infty}^{+\infty} \exp \left(-\frac{\left|x_{3}-\xi\right|}{r}\right) \frac{\partial\left[P_{S}^{2}-P_{1}^{2}(\xi)\right]}{\partial \xi} d \xi \\
& \times \frac{F_{24}(\alpha)}{2 a_{1}+\left[2 a_{12}+\left(Q_{66}^{2} / s_{66}\right)\right] P_{S}^{2}+2 a_{112} P_{S}^{4}} \\
& \times \frac{\left(Q_{12} s_{11}-Q_{11} s_{12}\right)}{s_{22}(\alpha) s_{11}-s_{12}^{2}} .
\end{aligned}
$$

Using an approximation for the $P_{1}$ profile (26),

$$
P_{1} \approx P_{S}\left[1-\exp \left(-\left|x_{3}\right| / R_{c}\right)\right] \operatorname{sgn}\left(x_{3}\right)
$$

we obtained from Eq. (15b) the following expression:

$$
\begin{aligned}
P_{2}\left(x_{3}\right) \approx & \frac{-f(\alpha) P_{S}^{2} p\left(x_{3}\right)}{2 a_{1}+\left[2 a_{12}+\left(Q_{66}^{2} / s_{66}\right)\right] P_{S}^{2}+2 a_{112} P_{S}^{4}}, \\
p\left(x_{3}\right)= & \frac{-2 R_{c}}{R_{c}^{2}-r^{2}}\left[\exp \left(-\frac{x_{3}}{R_{c}}\right)-\exp \left(-\frac{x_{3}}{r}\right)\right] \\
& +\frac{2 R_{c}}{R_{c}^{2}-4 r^{2}}\left[\exp \left(-\frac{2 x_{3}}{R_{c}}\right)-\exp \left(-\frac{x_{3}}{r}\right)\right] .
\end{aligned}
$$

Comparison of the approximate analytical solution (A5) with numerical calculations based on the coupled equations (17) is shown in Fig. 8. The difference between the approximate analytical expression (A5) (dashed curve) and numerical simulation (solid curve) is of the order of several percent. Thus, for $P_{2}\left(x_{3}\right)$, the analytical expression (A5) works with sufficient accuracy. *petr.yudin@epfl.ch

${ }^{1}$ L. Goncalves-Ferreira, S. Redfern, E. Artacho, and E. Salje, Phys. Rev. Lett. 101, 097602 (2008).

${ }^{2}$ A. K. Tagantsev, E. Courtens, and L. Arzel, Phys. Rev. B 64, 224107 (2001).

${ }^{3}$ V. Stepkova, P. Marton, and J. Hlinka, J. Phys.: Condens. Matter 24, 212201 (2012).

${ }^{4}$ D. A. Scrymgeour, V. Gopalan, A. Itagi, A. Saxena, and P. J. Swart, Phys. Rev. B 71, 184110 (2005).

${ }^{5}$ D. Lee, R. K. Behera, P. Wu, H. Xu, Y. L. Li, S. B. Sinnott, S. R. Phillpot, L. Q. Chen, and V. Gopalan, Phys. Rev. B 80, 060102 (2009).

${ }^{6}$ B. Houchmandzadeh, J. Lajzerowicz, and E. Salje, J. Phys.: Condens. Matter 3, 5163 (1991).

${ }^{7}$ V. Dvorak and V. Janovec, Jpn. J. Appl. Phys. 4, 400 (1965).

${ }^{8}$ E. A. Eliseev, A. N. Morozovska, G. S. Svechnikov, P. Maksymovych, and S. V. Kalinin, Phys. Rev. B 85, 045312 (2012).

${ }^{9}$ Note that unlike the common designation of $D_{1212} \equiv D_{1313} \equiv D_{2323}$ as $D_{44}$ in the crystallographic frame, in the laboratory frame with an inclined axis one has to distinguish $D_{1313} \equiv D_{55} \equiv D_{1212} \equiv D_{66}$ (independent on the angle $\alpha$ ) and $D_{2323} \equiv D_{44}$ with the latter depending on the inclination angle $\alpha$.

${ }^{10}$ N. A. Pertsev, A. G. Zembilgotov, and A. K. Tagantsev, Phys. Rev. Lett. 80, 1988 (1998).
${ }^{11}$ A. K. Tagantsev, L. E. Cross, and J. Fousek, Domains in Ferroic Crystals and Thin Films (Springer, New York, 2010), Chap. 6.2.2, pp. 300-304.

${ }^{12}$ L. Bulaevskii, Sov. Phys. Solid State 5, 2329 (1963).

${ }^{13}$ J. Axe, J. Harada, and G. Shirane, Phys. Rev. B 1, 1227 (1970).

${ }^{14}$ B. A. Strukov and A. P. Levanyuk, Ferroelectric Phenomena in Crystals (Springer, Berlin, 1998), Chaps. 3.2 and 10.2.

${ }^{15}$ N. A. Pertsev, A. K. Tagantsev, and N. Setter, Phys. Rev. B 61, R825 (2000).

${ }^{16}$ M. J. Haun, E. Furman, S. Jang, and L. Cross, Ferroelectrics 99, 45 (1989).

${ }^{17}$ N. A. Pertsev, V. G. Kukhar, H. Kohlstedt, and R. Waser, Phys. Rev. B 67, 054107 (2003).

${ }^{18}$ I. Ponomareva, A. K. Tagantsev, and L. Bellaiche, Phys. Rev. B 85, 104101 (2012).

${ }^{19}$ A. J. Bell, J. Appl. Phys. 89, 3907 (2001).

${ }^{20}$ P. Marton, I. Rychetsky, and J. Hlinka, Phys. Rev. B 81, 144125 (2010).

${ }^{21}$ G. Korn and T. Korn, Mathematical Handbook for Scientists and Engineers (McGraw-Hill, New York, 1961), Chap. 9.3.

${ }^{22}$ The equation $P-r^{2} \frac{d^{2} P}{d x^{2}}=E$ has a solution in the from $P(x)=$ $\frac{1}{2 r} \int_{-\infty}^{+\infty} \exp \left(-\frac{|x-\xi|}{r}\right) E(\xi) d \xi$. The only condition here is a vanishing solution at infinity. 